\section{Forever young - Der 60. Jahrgang der „Pneumologie“}

Forever Young - 60 Years of "Pneumologie“"
Mit dem Beginn des 60. Jahrgangs der „Pneumologie“ wechselt nach 5 Jahren erneut die Herausgeberschaft. Die Deutsche Gesellschaft für Pneumologie ist Herrn Professor Dr. Ulrich Costabel, der sich in den vergangenen Jahren mit großem Engagement und Erfolg der Aufgabe gewidmet hat, die „Pneumologie“ zu einer vorzüglichen pneumologischen Referenz weiterzuentwickeln, zu großem Dank verpflichtet. Unter der Leitung von Professor Costabel ist es gelungen, die Zeitschrift zu einem Forum für alle Pneumologinnen und Pneumologen in Deutschland zu machen, in der neben vorzüglicher Weiterbildung vor allem auch die breite Palette der wissenschaftlichen Bemühungen der deutschen Pneumologie in Originalien und Kasuistiken ihre Darstellung findet. Durch Ulrich Costabels langjährige Erfahrung mit der Herausgeberschaft des European Respiratory Journal hat auch die „Pneumologie“ bedeutend an Modernität und Professionalität gewonnen. Die Deutsche Gesellschaft für Pneumologie und der Thieme Verlag haben daher allen Grund, mit Stolz auf die von Professor Costabel geleistete Arbeit zurückzuschauen.

Mit Professor Costabel verlassen in diesem Jahr auch zwei bewährte und besonders effiziente Mitherausgeber das Team. Professor Dr. Detlef Kirsten und Professor Dr. Klaus Rabe haben wesentlichen Anteil an der bisherigen Erfolgsgeschichte der Zeitschrift. Wir danken ihnen namens der Deutschen Gesellschaft für Pneumologie und des Verlages für das Geleistete und hoffen von Herzen, dass sie der „Pneumologie“ auch in der Zukunft verbunden bleiben.
Mit dem Beginn des Jahres 2006 haben die Deutsche Gesellschaft für Pneumologie und der Thieme Verlag nun die Unterzeichner mit der Herausgeberschaft beauftragt, was wir als eine große Ehre, aber auch nicht geringe Herausforderung betrachten. Gemeinsam mit unseren „alten“ Mitherausgebern (Professor Dr. Claus Kroegel, Professor Dr. Joachim Müller-Quernheim) und den Neuen im Team (Professor Dr. Claus Vogelmeier und PD Dr. Hans-Werner Duchna) sind wir jedoch guten Mutes, der uns gestellten Aufgabe gerecht zu werden.

Zunächst betrachten wir es als unser Ziel, den hohen Standard des vorherigen Herausgeberteams zu halten. So werden wir uns weiterhin aufrichtig bemühen, in Editorials, Stellungnahmen und Serien zu bestimmten Themen sowie in den wissenschaftlichen Originalbeiträgen das ganze Spektrum der aktuellen pneumologischen Diskurse deutlich werden zu lassen. Auch werden weiterhin die zweimonatlichen CME-Fortbildungen in prägnanter Form Wissenswertes wiederholen und Gelegenheit zum Erwerb von Fortbildungspunkten geben. Fortführen wollen wir auch die Literaturreferate, damit den Leserinnen und Lesern ein rascher und prägnanter Überblick über die relevante internationale Publikationstätigkeit ermöglicht wird.

Ungeachtet unserer Verpflichtung zum Erhalt des Bewährten, haben aber auch wir einige neue Vorstellungen und Ideen, die dem Ziel dienen sollen, weiterhin eine attraktive und moderne deutschsprachige Fachzeitschrift zu produzieren. Nach unseren 
sehr kooperativen Gesprächen mit Herrn Peter Eich vom Thieme Verlag ist es uns gelungen, für das kommende Jahr einige praktische Neuerungen in Angriff zu nehmen. Zunächst werden wir im Laufe des Jahres ein elektronisches System der Manuskriptbearbeitung, wie es bei internationalen Zeitschriften üblich geworden ist, auch für die „Pneumologie“ einführen können. Dies erleichtert in hohem Maße das Einreichen eines Manuskripts und beschleunigt den Begutachtungsprozess. Weiterhin soll durch eine Änderung des Layouts die Lesbarkeit der publizierten Manuskripte durch eine noch klarer strukturierte Darstellungsweise sowie einen modernen Satz verbessert werden. Damit die Zeitschrift auch in den Weiten des Internet präsent ist, werden künftig alle Arbeiten der „Pneumologie“ zwei Jahre nach ihrem Erscheinen im Volltext kostenlos online verfügbar sein. Dies sind Schritte in die richtige Richtung, und wir danken dem Verlag hierfür!

Aber auch inhaltlich wollen wir neue Akzente setzen. Um die Erfolge der akademischen Pneumologie in Deutschland einer breiteren Öffentlichkeit zugänglich zu machen, haben wir die universitären Abteilungen gebeten, in den kommenden Monaten in einem kurzen Abriss über die Struktur, die wissenschaftlichen Schwerpunkte und die aktuell publizierten Arbeiten zu berichten. Dies kann in unseren Augen dazu dienen, die Vernetzung der akademischen pneumologischen Medizin mit der klinischen Pneumologie in Deutschland noch enger zu gestalten.

Selbstverständlich sind auch neue Serien in Angriff genommen worden. Die Vielzahl der neu entwickelten Medikamente in weiten Indikationsbereichen unseres Faches legt nahe, eine eigene Rubrik unter dem Titel „Pharmakologische Therapie“ einzurichten. In dieser soll ein unabhängiger und kritischer Überblick über Rationale, Wirkprinzip, Effektivität und unerwünschte Wirkungen neuer Pharmaka gegeben werden. Eine weitere neue Rubrik wird unter dem Titel „Pneumologisches Handwerkszeug“ Gelegenheit bieten, spezielle diagnostische oder therapeutische Technik im Detail kennenzulernen. Da in unserer bildträchtigen Zeit wichtige Informationen über besonders aussagekräftige und einprägsame Bilder transportiert werden, laden wir in einer neuen Serie dazu ein, entsprechende Bilder mit kurzer Beschreibung für die Rubrik „Pneumologische Bilder“ einzusenden. Schließlich wünschen wir uns, mehr Raum für sachliche Kontroversen in Form von „Pro und Contra“- oder „Standpunkt“-Beiträgen zu bieten.

Da auch unser Fach zurzeit erhebliche fachliche und politische Umbrüche erlebt und dabei den Strukturwandel der medizinischen Versorgung insgesamt nachvollzieht, werden wir versuchen, auch für die diese Prozesse reflektierende Diskussion ein Forum zu sein. Ein Schritt auf diesem Wege ist die Bemühung, den Berufsverband der Pneumologen, dessen Organ die „Pneumologie“ ja auch ist, deutlich sichtbarer werden zu lassen.

Bei allen oben geäußerten Plänen und Wünschen ist uns klar, dass diese sich nur umsetzen lassen, wenn sie als solche von den Leserinnen und Lesern mitgetragen werden. Wir laden Sie daher herzlich ein, sich an dem Leben Ihrer Zeitschrift mit Rat und Tat zu beteiligen.

In diesem Sinne hoffen wir, die Weichen für eine erfolgreiche Fortsetzung der Zeitschrift „Pneumologie“ gestellt zu haben, und freuen uns auf die bevorstehende Redaktionsarbeit.

Für das Team der „Pneumologie“

Ihre Herausgeber

Tom Schaberg und Santiago Ewig 